\title{
Sensornetzwerke für die Umwelt- und Geruchsüberwachung
}

\author{
Wolfhard Reimringer, Thorsten Conrad \\ 3S GmbH, Saarbrücken/D
}

\section{Zusammenfassung}

Sensornetzwerke bilden eine wesentliche Grundlage, schädliche Umwelteinflüsse räumlich engmaschig und nahezu zeitkontinuierlich zu überwachen. Dabei bestehen diese Netzwerke aus anwendungsspezifisch zusammengestellten und positionierten Sensorsystemen, die ihre Messdaten drahtlos an eine Servereinheit senden. Webbasierte Portale bieten Zugang zu den ausgewerteten, visualisierten und kartierten Daten. Anwendungen finden diese Netzwerke beispielsweise beim Monitoring von urbanen Gebieten (Straßenverkehr, Flug- und Seehäfen), bei der Steuerung der Dosierung von Abluftreinigungsanlagen und der Geruchsüberwachung in Wohngebieten.

\section{Einleitung}

Eine zeitlich und räumlich gut aufgelöste Überwachung unserer Umwelt ist die Grundlage für die Erkennung schädlicher Umwelteinflüsse und eine entsprechenden Reaktion, die die Gesundheit des Menschen in den Mittelpunkt stellt.

Quellen dieser Schad- und Geruchsstoffe sind dabei Verarbeitungsprozesse in der Industrie, beispielsweise in petrochemischen Produktionsanlagen, Klärwerken oder landwirtschaftlichen Betrieben. Ein wesentlicher Aspekt ist auch der Schadstoffausstoß durch den Straßenverkehr. In einschlägigen Gesetzestexten und Richtlinien [1, 2] sind bestimmte Schadstoffe wie beispielsweise PM10, Stickoxide und Schwefeloxide reguliert.

Die Schadstoffsituation wird aktuell mittels chemischer Analytik überwacht. Dabei greift man in der Praxis sowohl auf stationäre Analyseeinheiten als auch Passivsammler zurück, die in Einklang mit den gesetzlichen Bestimmungen die zeitlich mittlere Belastung bestimmen.

Bei Gerüchen orientiert man sich zurzeit an der Geruchsimmissions-Richtlinie (GIRL, [3]), die in Ergänzung zur TA Luft schädliche Umwelteinwirkungen durch Geruchsimmissionen behandelt. Im Rahmen dieser Richtlinie ist das Vorgehen zur Bewertung von
Geruchsbelästigungen durch menschliche Eindrücke einheitlich geregelt und wird im europäischen Ausland ebenfalls als QuasiStandard verwendet.

Ausgehend von analytischen Verfahren und der menschlichen Nase als "Ur-Sensor" für Gerüche sowie den normativen Regelungen gibt es schon seit einigen Jahren Ansätze, die Überwachung vieler Schad- und Geruchsstoffe in einer räumlich und zeitlich engmaschigen Weise über Sensoren durchzuführen. Dabei spielen Aspekte wie Kosten, Handhabung, Mobilität und die Limitationen der hierfür bereitstehenden Sensorik (Empfindlichkeit, Genauigkeit, Reproduzierbarkeit etc.) eine wesentliche Rolle.

Der vorliegende Beitrag gibt einen Überblick über die Funktionsweise von Sensorsystemen und -netzwerken sowie die Signalauswertung für die Umwelt- und Geruchsüberwachung und konzentriert sich dabei auf quasikontinuierliche, sensorbasierte Messverfahren und deren Einsatz im Feld.

\section{Sensoren und Sensorsysteme}

Die zur Messung von Schadstoffbelastungen und Geruchsbelästigungen eingesetzten Sensorsysteme verwenden meist die folgenden Sensortypen [4]:

- Metalloxid-Gassensoren 
- Elektrochemische Zellen

- NDIR

- PID

- Amperometrische Sensoren

Dabei werden als Sensorsysteme anwendungsspezifische Kombinationen der o. g. Prinzipien eingesetzt. Ergänzt werden diese "Primärsensoren" durch weitere Sensoren zur Bestimmung der Feuchte, der Außentemperatur, der Windrichtung, der Windgeschwindigkeit oder der generellen Umgebungssituation basierend auf Kamerasystemen, wobei letzteres in puncto Datenschutz nicht uneingeschränkt verwendbar ist.

Neben den sensorbasierten niedrig- und mittelpreisigen Lösungen bietet der Markt auch hochpreisige Verfahren wie beispielweise kompakte GC-MS-Systeme oder Systeme zur Ionenmobilitätsspektometrie, die nur in wenigen Spezialfällen im Sinne von Multiknotennetzwerken Anwendung finden.

Im Bereich der Geruchsdetektion dient in der Praxis durchaus auch die menschliche Nase als Sensor.

\section{Formierung von Netzwerken}

Ein Sensornetzwerk bezeichnet die Kombination einzelner Sensorsysteme (oder Sensorknoten) zu einem räumlich verteilten, Daten erfassenden Gesamtsystem.

Zentral für die Aussagekraft der Daten aus solchen Netzwerken ist die Anzahl der Stationen, deren passgenaue Bestückung mit Sensoren, deren Positionierung in Bezug auf die Kriterien wie Windrichtung, Topologie sowie dem Umstand, ob eine bekannte Quelle charakterisiert oder eine unbekannte Quelle lokalisiert werden soll.

Die einzelnen Sensorknoten sind bei heutigen Netzwerken zum Umweltmonitoring üblicherweise nicht direkt miteinander vernetzt, sondern mit einem zentralen Server verbunden, der dann das Sammeln und die strukturierte Ablage der Daten zusammen mit entsprechenden Metadaten übernimmt. Gerade im Bereich der Geruchsüberwachung, wo der menschliche Eindruck maßgebend ist, gibt es Ansätze durch Verwendung einer Software-App auf mobilen Endgeräten, die Rückmeldungen von Bürgern gleich als Metadaten auf den Server zu legen.

Je nach Positionierung kann die Kommunikation über Datennetze mangels Verfügbarkeit nicht gewährleistet werden, weshalb jeder Netzwerkknoten - auch zur redundanten Datenhaltung - eine on-boardSpeichermöglichkeit z. B. in Form von Festplatten oder SD-Karten benötigt [5].

Zur flexiblen Handhabung der Sensorsysteme sind diese im Bereich der diffusionsbasierten Messung zumeist mit Batterien ausgestattet. Bei gepumpten Systemen kombiniert mit ggf. beheizter oder verdünnender Probenahme wird auf Solarpanel mit einer Pufferbatterie oder direkt auf eine festinstallierte Spannungsversorgung zurückgegriffen, was die Positionierung einschränkt.

Gerade im urbanen Kontext werden auch Straßenbahnen oder Fahrräder herangezogen, um einerseits eine orts- und wetterunabhängige Spannungsversorgung zu gewährleisten, andererseits eine mobile, sensorische Erfassungsmöglichkeit der Luftqualität zu ermöglichen [6].

\section{Signalauswertung}

Bei der Signalauswertung unterscheidet man grundsätzlich zwischen der Quantifikation und der Zustandserkennung (qualitative Aussage, beispielsweise in Form einer Ampel).

Bei der Messung von Schadstoffen müssen einzelne Gaskomponenten quantitativ gemessen werden. Dies erfordert selektive Sensoren bzw. eine Charakterisierung und Kalibrierung auf Labor- oder Prüffeldbasis, die es erlaubt, Querempfindlichkeiten zu identifizieren und zu kompensieren. Die Sensorknoten geben die kompensierten Werte aus. 
Bei der Auswertung von Geruchsbelastungen hingegen stellt sich die Situation deutlich komplexer dar: Hier werden oft mehrere Sensoren in Form eines Arrays verwendet, oder auch Sensoren, die durch spezielle Betriebsmodi virtuell einen entsprechenden Datenraum aufweisen. Die erhaltenen Signale werden basierend auf Formmarkmalen mit Verfahren der multivariaten Datenanalyse ausgewertet und das System so an die zu erkennenden Gerüche angelernt. Im laufenden Betrieb werden dann auf Basis von Klassifikationsverfahren vorher angelernte Zustände identifiziert.

Die auf diese Weise ermittelten Mess- bzw. Ausgabewerte der einzelnen Sensorknoten werden nun - meist serverbasiert - gesammelt und in vielfältiger Weise verwendet: Einerseits dienen sie als Eingangsgröße zur Kartierung einer zu Immissionssituation und lassen in die Vergangenheit gerichtete Bewertungen auf Basis der archivierten Daten zu. Emissionsseitig erfasste Daten können aber auch einer Ausbreitungsrechnung zugeführt werden und eine Vorhersage der Schadstoffoder Geruchsbelastung ermöglichen. Der Vorteil dabei ist, dass durch einen in kurzen Abständen aktualisierten Messwert eine deutlich bessere Vorhersage getroffen werden kann als über die Eingabe einer zeitlich sporadischen durchgeführten Analyse oder Rasterbegehung. Immissionsseitig angeordnete Sensorknoten können als Stützstellen bzw. Verifikationspunkte der Ausbreitungsrechnung verwendet werden.

Der Zugang zu diesen Monitoringdaten oder Ausbreitungsvorhersagen wird in der Regel über spezielle Webportale, die je nach Anwendungsfall konfiguriert werden können (Getrennte Zugänge bspw. für Behörden, für Bürger, für die produzierenden Unternehmen).

Gerade im Bereich des Geruchs gestaltet sich die Kalibrierung bzw. das Anlernen solcher Sensorknoten schwierig. Im wesentlichen geschieht die Kalibrierung auf Basis olfaktometrischer Referenzmessungen, wobei zu beachten bleibt, dass die eingesetzten Sensoren keine "Geruchssensoren", sondern VOC-Sensoren sind - es besteht also die Möglichkeit, dass Gerüche und VOCs nicht in Korrelation gebracht werden können. Dies muss im Vorfeld einer Installation gesondert geprüft werden.

\section{Anwendungsbeispiele}

Die beschriebenen Sensorsysteme bzw. Sensornetzwerke können vielfältig eingesetzt werden. Anwendungen reichen von der Schadstoffüberwachung von $\mathrm{NO}_{x}$ sowie Ozon über die Überwachung von Filteranlagen bis hin zur Immissionsüberwachung bei petrochemischen Produktionsanlagen oder der Abwasserüberwachung und -behandlung durch Steuerung der dosierten Menge an Reinigungschemikalien.

Am Londoner Flughafen Heathrow ist ein Sensornetzwerk aus 40 einzelnen Knoten im Einsatz [7], um Kerosin-Leckagen aufzuspüren. Dabei führt ein rückgerechnetes Ausbreitungsmodell („back tracking“) zur Lokalisierung potentieller Quellen.

In Zürich wurden verschiedene Straßenbahnen mit Sensorsystemen zur Luftgütebestimmung (verkehrsbedingte Schadstoffe wir NOx oder Ozon) ausgestattet [6] und die Periodizität der „mobilen“ Sensorknoten ausgenutzt, um eine Schadstoffbelastung vorherzusagen.

In Saarbrücken (im stadtnahen Erholungs- und Wohngebiet „Warndt") wurden im Rahmen eines Pilotprojekts geruchsbasierte Immissionsbelastungen untersucht [8] mit dem Ziel, eine vermutete Quelle im Bereich der petrochemischen Produktion als Verursacher zu bestätigen. Hier kamen insgesamt 8 Stationen zum Einsatz.

In Bari ist man dabei, ein kombiniertes stationär-mobiles Monitoringnetzwerk aufzubauen (ca. 20 stationäre Knoten), das mit 
verschiedenen Sensoren (elektrochemischen Zellen, PID-Sensor, NDIR-Sensor, optischer Partikelzähler) ausgestattet ist [9]. Es werden luftqualitätsrelevante Gase wie $\mathrm{NO}_{2}, \mathrm{O}_{3}, \mathrm{CO}$, $\mathrm{SO}_{2}, \mathrm{CO}_{2}$, TVOC und PM gemessen mit dem Ziel, teures und aufwendig zu bedienendes Analyseequipment zu ersetzen.

Im Bereich der Klimaforschung werden bei der Erstellung und Verifikation grundlegender Modelle zum $\mathrm{CO}_{2}$-Austausch zwischen Boden und Atmosphäre bzw. Wasser und Atmosphäre räumlich und zeitlich gut aufgelöste Daten benötigt. NDIR-basierte Sensornetzwerke bilden hierzu durch entsprechende Erfassung von $\mathrm{CO}_{2}$-Flüssen in verschiedenen Szenarien eine wesentliche Grundlage [10].

\section{Fazit und Ausblick}

Gezielt bestückte Sensornetzwerke können Schadstoff- und Geruchsbelastungen erfassen und durch Verwendung webbasierter Softwareplattformen visualisieren, kartieren, archivieren und auf Basis von Ausbreitungsrechnungen vorhersagen. Damit wird eine wesentliche Grundlage zur Überwachung der Umwelt geschaffen, die im Kontext immer schärfer werdender Richtlinien und Gesetzte, auch in Fernost, unerlässlich wird.

Ein wesentliches Merkmal dieser Sensornetzwerke ist die Kombination von räumlich als auch zeitlich gut aufgelöster Messdaten, die gewisse Umweltüberwachungen trotz der reduzierteren Genauigkeit im Vergleich zu analytischen Instrumenten erst möglich werden lassen. Tragende Philosophie hierbei ist, dass eine Messung mit schlechterer Qualität an vielen gut gewählten Orten im Ergebnis besser ist als eine hochgenaue und teure Messung an wenigen nicht frei wählbaren Orten.

Technisch herrschen dieser Philosophie folgend zwei Trends vor. Ein Trend geht hin zu
„Big data“, d. h. Low-Cost-Equipment verbunden mit der Auswertung riesiger Datenbestände mittels statistischer Ansätze und Verfahren. Ein anderer Trend bildet den Mittelweg zwischen Low-Cost-Systemen und der Analytik, hier arbeiten verteilt installierte Systeme mit spezifischer Probenahme im Pumpbetrieb.

Für beide Trends ergeben sich aber noch offene Fragestellungen im Bereich der (Re-) Kalibrierung, die Thema weiterer Forschungen sind [11].

Dem regulatorischen Nachholbedarf im Bereich des Geruchsmonitorings auf Basis technischer Hilfsmittel wird mit der Mitte 2015 auf europäischer Ebene eingerichteten Arbeitsgruppe CEN/TC 264/WG 41 Rechnung getragen.

\section{Literatur}

[1] RICHTLINIE 2008/50/EG DES EUROPÄISCHEN PARLAMENTS UND DES RATES vom 21. Mai 2008 über Luftqualität und saubere Luft für Europa

[2] Erste Allgemeine Verwaltungsvorschrift zum Bundes-Immissionsschutzgesetz (Technische Anleitung zur Reinhaltung der Luft - TA Luft) vom 24. Juli 2002

[3] Geruchsimmissions-Richtlinie - GIRL - in der Fassung vom 29. Februar 2008 und einer Ergänzung vom 10. September 2008

[4] SPINELLE, L. et al.: Sensitivity of VOC Sensors for Air Quality Monitoring within the EURAMET Key-VOC project, Fourth Scientific Meeting EuNetAir, 2015-06-03 2015-06-05, Linkoping University, Linkoping, Sweden

[5] REIMRINGER, W. et al.: A Versatile Outdoor Platform for MOX Sensor Field Tests, International WG1-WG4 meeting on New Sensing Technologies and Modelling for Air Pollution Monitoring, Aveiro, Portugal, 14.-15. October 2014 
[6] HUEGLIN, $\mathrm{CH}$. Et al.: Extended performance analysis of a sensor unit for $\mathrm{O} 3$ and NO2 ans operation of a small static sensor network in Zurich, Fourth Scientific Meeting EuNetAir, 2015-06-03 2015-06-05, Linkoping, Sweden

[7] JONES, R. et al.: Environmental Measurements using low cost sensors: Latest results and future directions, Third Scientific Meeting EuNetAir, Istanbul, 3-5 December 2014

[8] REIMRINGER, W. et al.: MOX Sensor Platform in Outdoor Odor Nuisance Monitoring, Fourth Scientific Meeting EuNetAir, 2015-06-03 - 2015-06-05, Linkoping, Sweden
[9] SURIANO, D. et al: Stationary and Mobile Low-Cost Gas Sensor-Systems for Air Quality Monitoring Applications, Fourth Scientific Meeting EuNetAir, 2015-06-03 2015-06-05, Linkoping, Sweden

[10] BASTVIKEN, D. et al.: Technical Note: Cost-efficient approaches to measure carbon dioxide (CO2) fluxes and concentrations in terrestrial and aquatic environments using mini loggers, Biogeosciences, 12, 3849-3859, 2015

[11] SPINELLE, L. et al.: Field calibration of a cluster of low-cost available sensors for air quality monitoring. Part A: Ozone and nitrogen dioxide, Sensors and Actuators $B$ 215 (2015) 249-257 